\title{
Bayesian Evaluation of Three Serological Tests for Detecting Antibodies against Brucella spp. among Humans in the Northwestern Part of Ecuador
}

\author{
Jorge Ron-Román, ${ }^{1,2,3,4}$ Lenin Ron-Garrido, ${ }^{1}$ Emmanuel Abatih, ${ }^{3}$ Maritza Celi-Erazo, ${ }^{1}$ Laura Vizcaíno-Ordóñez, ${ }^{1}$ \\ Jaime Calva-Pacheco, , ${ }^{1,2,3,4,5}$ Pablo González-Andrade, ${ }^{1,2,3,4,5}$ Dirk Berkvens, ${ }^{3}$ Washington Benítez-Ortíz, ${ }^{1,2,3,4,5,6}$ \\ Jef Brandt, ${ }^{3}$ David Fretin, ${ }^{7}$ and Claude Saegerman ${ }^{2 *}$ \\ ${ }^{1}$ Centro Internacional de Zoonosis (CIZ), Universidad Central del Ecuador, Quito, Ecuador; ${ }^{2}$ Research Unit of Epidemiology and Risk \\ Analysis Applied to Veterinary Sciences (UREAR-ULiège), Fundamental and Applied Research for Animal and Health (FARAH) Center, \\ Faculty of Veterinary Medicine, University of Liege, Liège, Belgium; ${ }^{3}$ Department of Biomedical Sciences, Institute of Tropical Medicine, \\ Antwerp, Belgium; ${ }^{4}$ Departamento de Ciencias de la Vida y la Agricultura, Carrera de Ingeniería Agropecuaria, Universidad de las Fuerzas \\ Armadas - ESPE, Sangolquí, Ecuador; ${ }^{5}$ Facultad de Ciencias Químicas, Universidad Central del Ecuador, Quito, Ecuador; ${ }^{6}$ Facultad de \\ Medicina Veterinaria y Zootecnia, Universidad Central del Ecuador, Quito, Ecuador; ${ }^{7}$ Department of Bacteriology and Immunology, \\ Veterinary and Agrochemical Research Centre, Brussels, Belgium
}

\begin{abstract}
Brucellosis is an important but neglected zoonosis that causes serious economic losses both in livestock and human populations. The aim of the present study was to estimate the true prevalence of brucellosis together with diagnostic sensitivity and specificity of three serological tests in humans of the northwestern part of Ecuador using a Bayesian approach adjusted for the dependencies among the multiple tests to avoid any misinterpretation. In addition, the causal agent responsible for human brucellosis was also identified. Using a total of 3,733 samples collected from humans in this area between 2006 and 2008, the prevalence of human brucellosis and the diagnostic test characteristics of the Rose Bengal fast agglutination test (RBT), Wright's slow agglutination test with ethylenediaminetetraacetic acid disodium salt dehydrate (EDTA) (SAT-EDTA), and indirect ELISA (iELISA) were estimated using a Bayesian approach. The estimated true prevalence of human brucellosis was $1 \%$ (credibility interval: $0.4-1.6)$. The sensitivities of iELISA and RBT were higher than and similar $(95.1 \%$ and $95.0 \%$, respectively) to those of SAT-EDTA (60.8\%). Even though all tests indicated a high specificity (>99.0\%), the specificity of SAT-EDTA was highest (99.9\%). The circulating strain in this study area was identified to be Brucella abortus biotype 4 based on culture and microbiological characterization. The RBT and the iELISA are recommended for estimating the true prevalence of human brucellosis and/or for surveillance programs following their high sensitivities and specificities. The proposed strategy supports evidence-based medicine for clinicians and policy-makers to ensure appropriate preventive and control program of brucellosis worldwide.
\end{abstract}

\section{INTRODUCTION}

Brucellosis is an infectious and contagious disease caused by Gram-negative coccobacilli, which can survive in the cells of the immune system. It has a high tendency to cause chronic infections in multiple species including humans. ${ }^{1-3}$

In many countries, brucellosis is an important disease that causes serious economic losses in livestock production. ${ }^{4}$ In Ecuador, these losses are estimated at 5.5 million US\$ per year. ${ }^{5}$ In humans, this zoonosis causes mainly losses in working time and costs related to diagnosis and long treatment durations. ${ }^{6}$

Brucellosis is transmitted to man by direct contact with blood, placentas, fetuses, or vaginal secretions of infected animals and through consumption of products of animal origin, mainly raw milk, cheese, or yoghurt. The disease may show variable symptoms in humans with a tendency for recurrence, even though many times the infection is asymptomatic. ${ }^{7,8}$ The incubation period varies between 1 and 5 weeks, and Brucella infection may be asymptomatic or symptomatic. ${ }^{9}$ The disease can develop subclinically, acutely, or as a lasting condition with variable progress. ${ }^{10}$

Despite the fact that brucellosis is a notifiable disease in Ecuador until 2007, the true incidence of human cases remains largely unknown because of underreporting. According

\footnotetext{
*Address correspondence to Claude Saegerman, Research Unit of Epidemiology and Risk Analysis Applied to Veterinary Sciences (UREAR-ULiège), Fundamental and Applied Research for Animal and Health (FARAH) Center, Faculty of Veterinary Medicine, University of Liège, Quartier Vallée 2, Ave. de Cureghem 7A, B42, Liège B-4000, Belgium. E-mail: claude.saegerman@uliege.be
}

to the Ministry of Health (MSP), only 111 human cases were reported between 1990 and 2007, whereas the National Institute for Statistics and Census (INEC) registered 152 persons hospitalized because of brucellosis between 1995 and 2007, in a country with 12 million inhabitants. ${ }^{11}$

Despite the low sensitivity of culture, the definitive diagnosis in humans consists in detecting the presence of the bacteria by bacteriological examination. However, the high cost and the risk for human infection during in vitro cultures prevent this method from becoming the standard diagnostic tool. Therefore, common immunological assays such as the Rose Bengal fast agglutination test (RBT), Wright's slow agglutination test with EDTA (SAT-EDTA), and indirect ELISA (iELISA) based on the detection of antibodies against Brucella spp. have mostly been used in combination for a practical early diagnosis of the disease. Whereas the RBT detects the presence of $\operatorname{lgM}$ and $\lg 1$, the SAT-EDTA detects the presence of IgM and IgA and the iELISA detects both IgG1 and IgG2. ${ }^{12,13}$ Because of the fact, it is expected that tests are conditional dependent. ${ }^{13}$ Because each diagnostic assay has its characteristic sensitivity and specificity, which varies according to its detectable immunoglobulin isotype, the results of the multiple tests have usually been assumed to follow a multinomial distribution and a Bayesian approach used to simultaneously estimate the true prevalence and diagnostic sensitivity and specificity. ${ }^{14-16}$

An important con sideration in the evaluation of multiple diagnostic tests is whether or not the tests can be assumed conditionally independent of each other given the true disease status. It has been demonstrated that the assumption of conditional independence (CID) may lead to biased estimates for test characteristics if in fact the tests are conditionally 
dependent. ${ }^{17}$ Because RBT, SAT-EDTA, and iELISA are based on sera (detection of and considered to be conditionally dependent on each other), any estimation procedure should adjust for the dependencies among the tests. ${ }^{14,15}$

There is a lack of reliable information about the true prevalence of human brucellosis in Ecuador at the population level. Indeed, the aim of the present study was to estimate the true prevalence of human brucellosis together with diagnostic sensitivity and specificity of RBT, SAT-EDTA, and iELISA applied to humans in the northwestern part of Ecuador using a Bayesian approach. In addition, we aimed to isolate and characterize Brucella responsible for human infections using culture-based methods.

\section{METHODS}

Ethical considerations. The protocol was thoroughly reviewed and approved for ethics by the Bioethics Committee of the Biomedical Center, Central University of Ecuador. Before being included in the study, all participants provided informed written consent. For minors, parents/ guardians provided written consent on their behalf.

Description of the study and the study region. Between 2006 and 2008, a spatial cross-sectional survey was conducted, based on blood sampling of people from NW Ecuador together with an epidemiological survey of brucellosis. The target population for this study was rural community along the provinces selected and with a certain risk associated with animal management (Figure 1). After informed consent, a total of 3,733 blood samples were taken from persons inhabiting the high altitude or
Sierra provinces such as Carchi (649 samples), eastern Imbabura (497 samples), and eastern Pichincha (1,386 samples) and the coastal provinces such as Esmeraldas (195 samples), Manabí (377 samples), western Imbabura (535 samples), and western Pichincha (94 samples). Selection of the zones was based on their high prevalence of bovine brucellosis that is between $4.0 \%$ and $10.62 \%$ in the Sierra and between $5.88 \%$ and $10.62 \%$ in the Coast $^{5}$ and the occurrence of $41.30 \%$ (19 of 46) of the human cases, as reported by MSP between 1997 and 2007, and 51.97\% (79 of 152) of the hospitalized brucellosis patients, as reported by INEC in 2008. ${ }^{11}$ A map of the zones at risk of brucellosis and the study area is shown on Figure 1.

All the volunteer participants included in the study were first surveyed with a detailed questionnaire at the same time of the blood culture sampling. This questionnaire was developed attending technical and ethical consideration to register the following information: personal identification (first name, family name, address, contact details, and phone number if possible), basic demographic data (age, gender, province of birth, and province of residence), professional and/or occupational activities and development period of such activity, contact with species of livestock interest (cattle, sheep, pigs, goats, and horses), contact with fetuses, aborted products, and/or secretions of animals of livestock interest and eating habits (consumption of milk, cheese, yogurt, livestock fresh blood and viscera, fetuses, and bovine placenta). In addition, a section of the survey was designed to collect information on the following symptoms detected by the participant itself during the last 12 months before the date of the blood sampling: fever, shaking chills, weakness, night sweats, joint

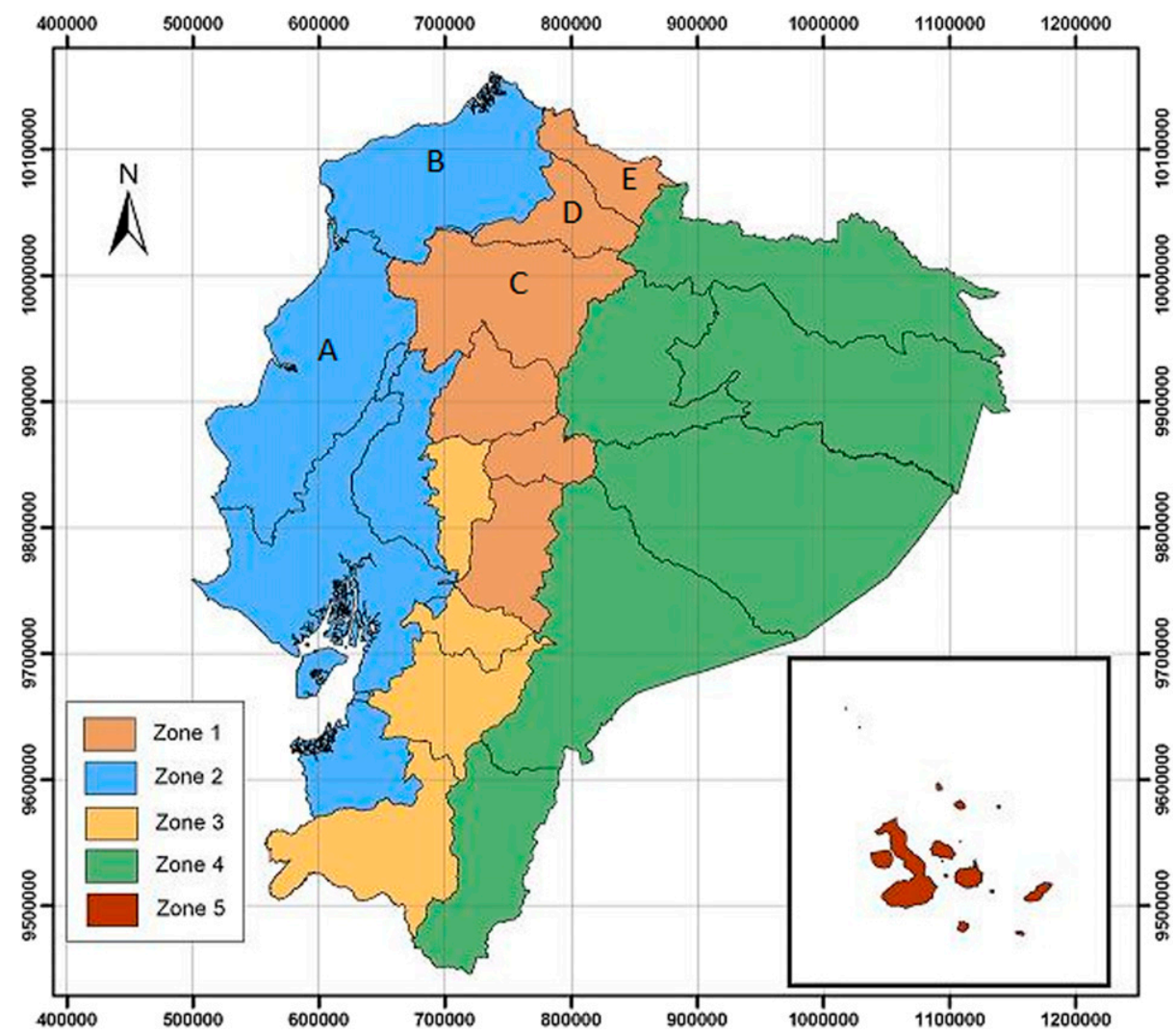

FiguRE 1. Zones at risk of brucellosis in Ecuador and the location of the study area. Zones at risk of brucellosis in Ecuador: Zone 1, high prevalence zone on Sierra; Zone 2, high prevalence zone in coastal area; Zone 3, low prevalence zone on Sierra; Zone 4, presumed low prevalence zone in Amazonia; and Zone 5, presumed brucellosis-free zone of the Galapagos Islands. Location of the study area (provinces): A, Manabi; B, Esmeraldas; C, Pinchincha; D, Imbabura; and E, Carchi. This figure appears in color at www.ajtmh.org. 
pains, muscles pains, headaches, allergies, insomnia, and anorexia. Data collected were then correlated with the serological test results and were carefully analyzed, especially in those cases of participants with at least one of the tests showing positive results $(n=104)$.

Diagnostic assays. Three serological assays to detect antibodies against Brucella spp. were used: RBT, SAT-EDTA, and iELISA. ${ }^{18}$ The protocols are available in Supplemental Appendix A. Samples were processed and analyzed in the laboratory for immunodiagnosis at the International Centre for Zoonoses (ClZ) of the Central University of Ecuador.

Isolation and typing of Brucella spp. Isolation of the causal agent was reached by blood culture using the automated system BACTEC (Becton Dickinson Diagnostic Instrument Systems, Sparks, MD) facilitated by the Microbiology laboratory of the "Hospital Vozandes." Not all the sampled population in this study was submitted to blood culture process. Thus, selection of acute cases to make the most of the possibilities of getting the causal agent isolated was carried out after careful analysis of the serological assay results previously performed. As follows, a number of participants $(n=22)$ showing positive results for RBT and elevated titers of SAT-EDTA were contacted and sampled for a blood culture tests on their location. For logistical reasons concerning the suitable and almost immediate transportation of blood culture samples until the "Hospital Vozandes" in Quito city, only participants located in the highlands were selected. None of them was a minor ( $<18$ years old) at the moment of sampling.

For each participant, a set of three bottles were sampled for blood culture testing following internal procedures stated at $\mathrm{CIZ}$. Briefly, the set consisting of three bottles was obtained by three different punctures separated by 30 minutes each. Ten milliliters of venous blood was taken using a syringe from the cephalic vein and then inoculated into the blood culture bottle after changing a new sterile needle. The bottles were then maintained into a plastic thermic box at room temperature $\left(18-25^{\circ} \mathrm{C}\right)$ and then transported as fast as was possible to the "Hospital Vozandes" laboratory.

Each bottle was incubated into the BACTEC system until positive bacterial growth was detected or for a maximum period of 30 days to be considered as negative. Isolates were identified as Brucella sp. following the internal procedures stated in the hospital. Then, the species and biotype were determined based on the following assays performed at $\mathrm{ClZ}$ and the Veterinary and Agrochemical Research Centre: 1) macroscopic and microscopic observation of the colonies in purified culture plates, 2) biochemical assays (oxydase, catalase, and urease), 3) production of $\mathrm{H} 2 \mathrm{~S}, 4) \mathrm{CO} 2$ growth requirement, 5) growth in stained media (thionine, basic fuchsin, and safranin), 6) agglutination with monospecific sera $A$ and $M$, and 7) performance of polymerase chain reaction (PCR)-bscp31 (i) gene and PCRAbortus, Melitensis, Ovis and Suis (AMOS) (ii). The two PCRs were chosen from the amplified regions of two different Brucella genes: 1) primers B4 (5P-TGG CTC GGT TGC CAA TAT CAA3P) and B5 (5P-CGC GCT TGC CTT TCA GGT CTG-3P) amplified a 223-bp fragment present on a gene encoding a 31-kDa Brucella abortus antigen ${ }^{19,20}$ 2) a 498-bp fragment was amplified with AMOS PCR, which was derived from IS711 gene. ${ }^{21}$

Statistical analysis. Apparent prevalence and correlation analysis. The apparent prevalence was calculated for the three separate tests and for the combined tests using a parallel interpretation, that is, a person is considered positive if one or more tests yielded a positive result. The concordance observed was computed between the results of the respective tests, and they were plotted. This concordance observed was obtained counting the concordance between positive and negative in two tests with respect to the total samples.

Model building and diagnostics. Using the cross-classified test results for the three tests, it was assumed that the resulting cell frequencies/counts followed a multinomial distribution. Using the models developed by Branscum et al. ${ }^{15}$ and Berkvens et al., ${ }^{16}$ the corresponding multinomial cell frequencies were expressed in terms of the true prevalence of human brucellosis in the population, the sensitivities, and the specificities of RBT, SAT-EDTA, and iELISA and additional terms to account for the conditional dependence (CD) between each pair of tests (Supplemental Appendix B). In this scenario, including all parameters and possible interactions between the three individual tests requires 15 parameters to be estimated, namely, the prevalence, the sensitivity, and the specificity of the first test; two conditional sensitivities and two conditional specificities for the second test; and finally, four conditional sensitivities and four conditional specificities for the third test. This model is in fact non-identifiable because the data only allow seven parameters to be estimated. As none of the three tests is considered a gold standard test and the tests are not conditionally independent, constraints have to be imposed on a subset of the parameters to reduce the number of parameters to be estimated. ${ }^{16}$

By combining the results of the applied assays (RBT, SATEDTA, and iELISA), the prevalence of this zoonosis among humans in the NW Ecuador was estimated together with the sensitivity and the specificity of each of the tests. To evaluate the goodness of fit of the models, the Bayesian $P$-value, deviance information criterion (DIC), ${ }^{22}$ and the number of effective parameters estimated $(p D)^{16}$ were used as calibrating parameters. Briefly, the DIC ensures that the most parsimonious model is used. It is calculated as $\mathrm{DIC}=p D+D$, with $D$ the mean posterior deviance and $p D$ the number of parameters effectively estimated by the model. Models with a smaller DIC should be preferred over models with a larger DIC. The Bayesian $P$-value is a posterior predictive check that detects lack of fit of the model to the data. It is based on the difference between the deviance of the observations and the deviance of observations generated randomly from the presently fitted model and should have a value around 0.50 . The values of $p D$ and DIC evaluated based on the posterior means of the multinomial probabilities and those based on the posterior means of the parameters should be as close as possible. ${ }^{16}$

Modeling CD. Using the model that assumes CID among the three tests, given the true disease status of individuals as the baseline model, CD (i.e., $P\left(T_{2}^{+} \cap T_{1}^{+} \mid D^{+}\right) \neq P\left(T_{2}^{+} \cap T_{1}^{-} \mid D^{+}\right)$, $P\left(T_{2}^{-} \cap T_{1}^{-} \mid D^{-}\right) \neq P\left(T_{2}^{-} \cap T_{1}^{+} \mid D^{-}\right)$, where $T_{1}$ or $T_{2}$ are the positive or negative results for test 1 or test 2 and $D$ is the individual disease status [+] or [-]) between each pair of tests was estimated using different parameterizations of the model that assumed CD between tests. ${ }^{15,16,23}$ Essentially, in the first parameterization, the CD between IELISA and RBT was modeled and the data used were based on cross-classified test results with iELISA on the first column, RBT on the second column, and SAT-EDTA on the third column (Table 1).

The remaining two parameterizations involving the $C D$ between two tests were obtained by switching columns for iELISA with RBT and SAT-EDTA in turn. In addition, three models were constructed with $C D$ between the pairs: iELISA/RBT and iELISA/SAT-EDTA, iELISA/RBT and 
RBT/SAT-EDTA, and between iELISA/SAT-EDTA and RBT/SAT-EDTA, respectively. ${ }^{23}$ Finally, a model with CD among all the three tests was considered (all pairs inclusive) separately among infected and noninfected individuals and combined.

The models can be summarized as follows:

- Model 1: CID

- Model 2: CD between RBT and SAT-EDTA

- Model 3: CD between iELISA and RBT

- Model 4: CD between iELISA and SAT-EDTA

- Model 5: CD between iELISA and SAT-EDTA and between RBT and SAT-EDTA

- Model 6: CD between iELISA and RBT and between iELISA and SAT-EDTA

- Model 7: CD between iELISA and SAT-EDTA and between RBT and SAT-EDTA

- Model 8: CD among all tests for infected subjects that are between iELISA and SAT-EDTA, between iELISA and RBT, and between RBT and SAT-EDTA

- Model 9: CD among all tests for noninfected subjects that are between IELISA and SAT-EDTA, between iELISA and RBT, and between RBT and SAT-EDTA

- Model 10: CD among all tests that are between iELISA and SAT-EDTA, between iELISA and RBT, and between RBT and SAT-EDTA

Representing the CD between pairs of tests among infected humans by $a$ and among the noninfected population by $b$, posterior estimates were obtained along with their $95 \%$ credibility intervals (Cr. Is.). If the $\mathrm{Cr}$. I. includes zero, it will imply that the data do not provide enough evidence against the null hypothesis of CID, whereas if the interval excludes zero, the CID assumption between the two tests will be rejected in favor of the alternative hypothesis of $C D$. All models were compared using the DIC. To be considered significantly different, the reduction in DIC between any two models should be more than three units. In situations where the difference in DIC was smaller than three units, the models were assumed to be similar and selection was based on parsimony. ${ }^{22}$

Prior distributions for parameters. In Ecuador, the prevalence, sensitivities, and specificities of the three tests used are not known. Priors were therefore introduced based on similar studies among humans in different countries found in the literature. The average prevalence of brucellosis among humans ranged between 0 and $0.2,{ }^{24,25}$ and sensitivity and specificity ranged between 0.88 and 1 and between 0.93 and

TABLE 1

Cross-classified test results for human brucellosis in cattle in the northwestern part of Ecuador based on RBT, SAT-EDTA, and iELISA

\begin{tabular}{cccr}
\hline RBT & SAT-EDTA & iELISA & Number \\
\hline 0 & 0 & 0 & 3,663 \\
0 & 0 & 1 & 7 \\
0 & 1 & 0 & 0 \\
0 & 1 & 1 & 2 \\
1 & 0 & 0 & 11 \\
1 & 0 & 1 & 31 \\
1 & 1 & 0 & 1 \\
1 & 1 & 1 & 18 \\
Total & - & - & 3,733 \\
\hline
\end{tabular}

A test with $\mathrm{EDTA} ; 1=$ positive; $0=$ negative
1, respectively, for iELISA; 0.49 and 1 and between 0.97 and 1 for SAT-EDTA, respectively; and last, 0.9 and 1 and 0.95 and 1 , respectively, for RBT. ${ }^{2,26-28}$ Lower and upper limits of these ranges were used to define uniform distributions for the parameters.

Prior information on the eight covariance parameters (four for infected and four for the noninfected individuals) were not available, so their ranges were generated based on priors for the sensitivities and specificities for the three tests (see Supplemental Appendix B). Uniform prior distributions were then assumed based on the ranges. ${ }^{15}$

Model diagnostics. All models were run using three chains, a burn-in period of 10,000 iterations, and another 20,000 iterations (with thinning applied) to obtain the posterior estimates. Trace plots were simultaneously combined with autocorrelation plots to explore the convergence of the model. A more formal test for convergence, the Brooks, Gelman, and Rubin (BGR) convergence statistic was used to assess model convergence, and only properly converged models were further considered. ${ }^{29}$ The WinBugs code used is found in Supplemental Appendix B.

Sensitivity analyses. Several studies have indicated that for the CD model, the prior information influences the posterior estimates. ${ }^{15,30,31}$ The influence of prior information on the posterior estimates was assessed by conducting a sensitivity analysis. This was performed by using non-informative priors (NIPs) and slight perturbations (in steps of $10 \%$ or $15 \%$ ) of the prior intervals. ${ }^{30}$ The following sets of priors were considered:

- NIP for the prevalence and information priors (IPs) for the sensitivities and specificities

- NIP for the prevalence and for the sensitivities and IPs for the specificities

- NIP for the prevalence and for the specificities and IPs for the sensitivities

- IP for the prevalence and NIPs for the sensitivities and specificities

- IP for the prevalence and for the sensitivities and NIPs for the specificities

- IP for the prevalence and for the specificities and NIPs for the sensitivities

- Perturbations of the prior interval

For each set of alternative prior distributions considered for the parameters, the model was run with the same number of chains and similar diagnostics were performed. It is also known that inferences based on multinomial data with zero cell frequencies may lead to slow convergence or nonconvergence, and hence lead to inaccurate results, especially when the total sample size is small. ${ }^{32}$ Because there are no immediate corrections or exact methods, the effects of zero-multinomial frequencies on the estimates were evaluated by replacing each zero frequency with a one. Replacing zero frequencies by ones has a minimum effect on the balance prior information versus observed data, but helps convergence, and hence assists to evaluate model consistency/robustness.

\section{RESULTS}

Apparent prevalence and correlation analysis. The cross-classified test results of the three serological tests on the 3,733 samples are shown on Table 1. The apparent prevalence was $0.56 \%(95 \% \mathrm{Cl}: 0.35-0.85)$ based on SATEDTA, $1.63 \%$ (95\% Cl: 1.25-2.09) based on RBT, and $1.55 \%$ (95\% Cl: $1.18-2.00)$ based on iELISA. The prevalence of 
brucellosis based on the three tests was 1.88\% (1.46-2.36), following a parallel interpretation. The prevalence based on RBT was observed to be similar to that obtained based on iELISA. A correlation analysis yielded a high observed concordance of 0.96 between the results obtained based on RBT (Test 1) and iELISA (Test 3). In addition, between RBT (Test 1) and SAT-EDTA (Test 2) and between SAT-EDTA (Test 2) and iELISA (Test 3), the observed concordances also appeared to be high (0.97 and 0.96, respectively).

Model selection. The estimated DIC, $p D$, and Bayesian $P$-values for the different models are presented in Table 2. A remarkable observation from the models was the closeness of the Bayesian $P$-values to 0.5 (values ranged between 0.4817 and 0.5636 ), indicating that all the models we tested provided acceptable fit to the data. All the DIC values were fairly close to each other with differences smaller than three. Using the CID model as the baseline model (DIC $=34.230)$, the only other model that yielded a slightly higher reduction in DIC than the baseline model was Model 9 (DIC = 31.955); the model with CD among the three tests for noninfected subjects (Table 2). Because of the borderline significance of the difference in DIC (difference $=2.3$ ), the selection of the most appropriate model was based on parsimony and completeness (the fewer the number, the better the estimated parameters). Model 9 appeared to be more parsimonious because its $p D, 4.376$, is slightly lower than that of model $10(p D=4.597)$ (Table 3). The final model was therefore based on the model with CD among the three tests for noninfected individuals (Model 9).

For this model, an observation of the trace plots indicated that the chains were mixing properly. However, the autocorrelation plots indicated that there were significant autocorrelations up until lag 40. On thinning with a thinning coefficient of 60 , we ensured that the samples were independent. The BGR plots indicated no problems with convergence. In addition, the estimated Bayesian $P$-value for the final model was 0.4865 further corroborating the aforementioned findings that the model provides a good fit to the data. The estimated $p D$ and DIC were 4.376 and 31.955, respectively. These values in turn appear to be reasonably close to the optimal values (5.505 and 35.972 , respectively) further supporting the claim that the model fits the data well.
True prevalence, accuracy, and CD. The posterior mean estimates of the prevalence, the sensitivity, the specificity, and the dependency coefficients for the three tests among noninfected subjects are shown in Table 3. According to the results, the true prevalence of brucellosis among humans was $1.0 \%$ (95\% Cr. I.: $0.40-1.60)$. The performances of iELISA and RBT were very similar with estimated sensitivities and specificities of 95.1\% (88.7-99.8) and 95.0\% (90.3-99.7) and $99.3 \%$ (98.7-99.8) and 99.2\% (98.6-99.7), respectively. Wright's slow agglutination test with EDTA had the lowest sensitivity of $60.8 \%$ (40.7-96.6) and the highest specificity of 99.9\% (99.8-1).

The $95 \%$ probability intervals for the conditional dependency coefficients among the three tests for the noninfected humans excluded zero, implying that the hypothesis of CID among the three tests is rejected in favor of the alternative hypothesis of CD. The highest CD coefficient was observed between the specificities of iELISA and RBT (0.005), whereas a lower CD was observed between iELISA and SAT-EDTA and between SAT-EDTA and RBT (0.0004) (Table 3).

Sensitivity analysis. When a NIP was used for the prevalence and IPs used for the sensitivities and specificities, or IPS used for the specificities and NIPs used for the sensitivities, the results were quite similar. On the other hand, when NIPs were used for the prevalence and the sensitivities and IPs were used for the specificities, there were slight but nonsignificant differences in the estimated prevalence and specificities. Similarly, when using an IP for the prevalence and NIPs for the sensitivities and specificities, or IPs used for the specificities and NIPs were used for the sensitivities, there were slight but nonsignificant differences (Cr. Is. overlap) in the estimated prevalence and sensitivities. The results were quite similar when IPs was used for the prevalence and the sensitivities and NIPs were used for the specificities.

Decreasing the lower limits of all the prior intervals by $10 \%$ had no influence on the estimated parameter values and their 95\% Cr. I. However, when both the lower and upper limits of the prior interval were decreased by $10 \%$, the model did not converge $(p D=2.57$ and $\mathrm{DIC}=688.54)$. In addition, the Bayesian $P$-value was 1 , indicating a considerable lack of fit of the data to the model.

TABLE 2

Deviance information criterion (DIC), number of estimated parameters $(p D)$, and Bayesian $P$-values for the model assuming conditional independence (CID), models assuming condition dependence between each of two tests, models including pairs of conditional dependence (CD), and a model with condition dependence between all the three tests

\begin{tabular}{|c|c|c|c|}
\hline Type of model & $\mathrm{DIC}$ & $p D$ & Bayesian $P$-value \\
\hline Model 1: CID model & 34.230 & 3.822 & 0.5357 \\
\hline Model 2: CD between RBT and SAT-EDTA among all subjects & 34.666 & 4.359 & 0.5354 \\
\hline Model 3: CD between iELISA and RBT among all subjects & 34.610 & 4.888 & 0.4887 \\
\hline Model 4: CD between iELISA and SAT-EDTA among all subjects & 34.472 & 4.259 & 0.5357 \\
\hline $\begin{array}{l}\text { Model 5: CD: between iELISA and RBT and between SAT-EDTA and RBT } \\
\text { among all subjects }\end{array}$ & 34.480 & 4.513 & 0.5021 \\
\hline $\begin{array}{l}\text { Model 6: CD: between iELISA and RBT and between iELISA and SAT-EDTA } \\
\text { among all subjects }\end{array}$ & 34.437 & 4.690 & 0.4817 \\
\hline $\begin{array}{l}\text { Model 7: CD: between iELISA and SAT-EDTA and between RBT and SAT-EDTA } \\
\text { among all subjects }\end{array}$ & 32.664 & 4.226 & 0.5636 \\
\hline $\begin{array}{l}\text { Model 8: CD: between iELISA and SAT-EDTA and between RBT and SAT-EDTA } \\
\text { among infected subjects }\end{array}$ & 35.563 & 4.525 & 0.5515 \\
\hline $\begin{array}{l}\text { Model 9: CD: between iELISA and SAT-EDTA and between RBT and SAT-EDTA } \\
\text { among noninfected subjects }\end{array}$ & 31.955 & 4.376 & 0.4865 \\
\hline $\begin{array}{l}\text { Model 10: CD: between iELISA, SAT-EDTA and between iELISA and RBT and } \\
\text { between RBT and SAT-EDTA among all subjects }\end{array}$ & 32.007 & 4.597 & 0.5059 \\
\hline
\end{tabular}


TABLE 3

Posterior mean estimates and 95\% credibility intervals (Cr. I.) of the prevalence, sensitivities (Se), and specificities (Sp) of iELISA, SAT-EDTA, and RBT and conditional dependence (CD) coefficients between the three tests among noninfected subjects

\begin{tabular}{|c|c|c|c|c|}
\hline & & & Model 1 & Model 9 \\
\hline Test & Parameter & Prior & Posterior mean (Cr. I.) & Posterior mean (Cr. I.) \\
\hline \multirow[t]{2}{*}{ RBT } & $\mathrm{Se}$ & Uniform (0.90-1) & $0.936(0.902-0.981)$ & $0.950(0.903-0.997)$ \\
\hline & $\mathrm{Sp}$ & Uniform $(0.95-1)$ & 0.997 (0.995-0.999) & $0.992(0.986-0.997)$ \\
\hline \multirow[t]{2}{*}{ SAT-EDTA } & $\mathrm{Se}$ & Uniform $(0.40-1)$ & $0.446(0.402-0.537)$ & $0.608(0.407-0.966)$ \\
\hline & $\mathrm{Sp}$ & Uniform $(0.99-1)$ & $0.999(0.999-1.000)$ & $0.999(0.998-1)$ \\
\hline \multirow[t]{3}{*}{ iELISA } & $\mathrm{Se}$ & Uniform $(0.88-1)$ & $0.942(0.885-0.991)$ & $0.951(0.887-0.998)$ \\
\hline & $\mathrm{Sp}$ & Uniform (0.93-1) & $0.998(0.998-0.999)$ & $0.993(0.987-0.998)$ \\
\hline & Prevalence & Uniform (0-1) & $0.015(0.011-0.020)$ & $0.010(0.004-0.016)$ \\
\hline ¡ELISA and SAT-EDTA & $\mathrm{CD}$ & - & - & $0.0004(0.00001-0.00156)$ \\
\hline iELISA and RBT & $C D$ & - & - & $0.0046(0.00022-0.00956)$ \\
\hline \multirow[t]{4}{*}{ RBT and SAT-EDTA } & $\mathrm{CD}$ & - & - & $0.0004(0.00001-0.00147)$ \\
\hline & DIC & - & 34.230 & 31.916 \\
\hline & $p D$ & - & 3.822 & 4.372 \\
\hline & Bayesp & - & 0.5357 & 0.4817 \\
\hline
\end{tabular}

Bayesp = Bayesian P-values; DIC = Deviance information criterion; iELISA = indirect ELISA; $p D=$ number of estimated parameters; RBT = Rose Bengal test; SAT-EDTA = Wright's slow agglutination test with EDTA.

Finally, replacing the zero-observed frequencies with one and running all the different models, none of the models appeared to be doing better than the CID model. However, the model with CD between the specificities yielded a DIC that was closest to that of the CID model (data not shown). Based on this model, the estimated prevalence, sensitivities, specificities, and dependence coefficients were quite similar (Cr. Is. overlap, data not shown).

Isolation and characterization of circulating Brucella spp. in Northwest Ecuador. From three positive cases (of 22 tested), B. abortus biotype 4 was isolated. Blood cultures were only positive for patients with higher levels of IgM antibodies (SAT-EDTA). Detailed information about these three persons with positive blood cultures (as well as the culture-negative persons) is given in Table 4 with the characteristics of the isolations, bacteriological data, and PCR in Table 5 and Figures 2 and 3, respectively. In Figure 2, the gel of agarose for electrophoresis shows the amplification of the bscp31 gene; Lane MP (molecular weight marker): $100 \mathrm{bp}$ DNA Ladder and Lane
$\mathrm{H} 1-\mathrm{H} 3$ of human strains. In Figure 3, the 498-bp fragment was amplified with AMOS PCR, which was derived from IS711 gene.

The results of the retrospective questionnaire, administered to the seropositive persons relating to possible symptoms (in the last year) relevant for brucellosis, are presented in Table 6.

\section{DISCUSSION}

Given that the main objective of an epidemiological survey on human brucellosis should include the identification of brucellosis cases together with the determination of the source of infection that is occupational or alimentary, ${ }^{33}$ the present study aimed to provide a reliable estimate of the prevalence based on the detection of antibodies against Brucella spp., and the isolation and identification of the causal agent among humans in the NW Ecuador.

The estimation of the true prevalence and diagnostic test characteristics was performed using a Bayesian approach in the absence of a standard reference test and with no

TABLE 4

Results of blood cultures from patients with high serological titers concomitant with brucellosis presumptive clinical symptoms (northwest Ecuador)

\begin{tabular}{|c|c|c|c|c|c|c|c|c|c|}
\hline $\mathrm{N}^{\circ}$ & ID & Sample code & Age & Gender & Occupation & $\mathrm{RB}$ & SAT-EDTA (Ul/mL) & iELISA (U/mL) & Blood culture \\
\hline 1 & 93 & SHB-Ma-93 & 28 & $\mathrm{M}$ & Farmer & + & - & 60 & - \\
\hline 2 & 104 & SHB-Ma-104 & 22 & $\mathrm{M}$ & Farmer & + & 100 & 60 & - \\
\hline 3 & 146 & SHB-Ma-146 & 17 & $\mathrm{M}$ & Student & + & 80 & 60 & - \\
\hline 4 & 187 & SHB-Ma-187 & 49 & $\mathrm{~F}$ & Farmer & - & 80 & - & - \\
\hline 5 & 198 & SHB-Ma-198 & 28 & $M$ & Farmer & - & - & 30 & - \\
\hline 6 & 325 & SHB-Ma-325 & 49 & $\mathrm{~F}$ & Farmer & + & 100 & 60 & - \\
\hline 7 & 344 & SHB-Ma-344 & 50 & $M$ & Farmer & - & 50 & - & - \\
\hline 8 & 372 & SHB-Ma-372 & 39 & $M$ & Farmer & + & 100 & 60 & - \\
\hline 9 & 820 & SHB-IA-1 & 41 & $\mathrm{M}$ & Veterinary lecturer & + & 80 & 60 & - \\
\hline 10 & 821 & SHB-IA-2 & 26 & $\mathrm{M}$ & Farmer & + & 100 & 60 & - \\
\hline 11 & 822 & SHB-IA-3 & 39 & $\mathrm{M}$ & Farmer & + & 100 & 60 & - \\
\hline 12 & 825 & SHB-IA-6 & 41 & $\mathrm{M}$ & Farmer & - & 100 & 60 & - \\
\hline 13 & 2,202 & SHB-Cam-Nor-5 & 39 & $\mathrm{~F}$ & Slaughterhouse worker & + & 50 & 60 & - \\
\hline 14 & 2,330 & SHB-Cam-Nor-133 & 42 & $\mathrm{M}$ & Slaughterhouse worker & + & 40 & 60 & - \\
\hline 15 & 2,331 & SHB-Cam-Nor-134 & 55 & $\mathrm{M}$ & Transporter & + & - & 50 & - \\
\hline 16 & 2,343 & SHB-Cam-Nor-146 & 50 & $\mathrm{~F}$ & Slaughterhouse worker & + & - & 48.6 & - \\
\hline 17 & 2,348 & SHB-Cam-Nor-151 & 66 & $\mathrm{~F}$ & Slaughterhouse worker & + & 60 & 60 & - \\
\hline 18 & 2,349 & SHB-Cam-Nor-152 & 36 & $\mathrm{~F}$ & Slaughterhouse worker & + & 320 & 14.4 & + \\
\hline 19 & 2,353 & SHB-Cam-Nor-156 & 35 & $\mathrm{M}$ & Slaughterhouse worker & + & 100 & 26.2 & - \\
\hline 20 & 2,356 & SHB-Ay-10 & 27 & $\mathrm{M}$ & Veterinary student & + & 1,600 & 60 & + \\
\hline 21 & 3,144 & SHB-Zon-Nor-370 & 58 & $M$ & Farmer & + & 800 & 60 & + \\
\hline 22 & 3,409 & SHB-Zon-Nor-635 & 21 & $\mathrm{M}$ & Farmer & + & 960 & 60 & - \\
\hline
\end{tabular}

ID = identification number; iELISA = indirect ELISA; RBT = Rose Bengal test; SAT-EDTA = Wright's slow agglutination test with EDTA. 
TABLE 5

In vitro characteristics of the isolations

\begin{tabular}{|c|c|c|c|c|c|c|c|c|c|c|}
\hline \multirow[b]{2}{*}{ Sample code serology } & \multirow{2}{*}{$\begin{array}{l}\text { Sample code } \\
\text { bacteriology }\end{array}$} & \multirow{2}{*}{$\begin{array}{l}\text { Urease } \\
\text { activity }\end{array}$} & \multirow{2}{*}{$\begin{array}{l}\mathrm{CO}_{2} \text { for } \\
\text { growth }\end{array}$} & \multirow{2}{*}{$\begin{array}{c}\mathrm{H}_{2} \mathrm{~S} \\
\text { production }\end{array}$} & \multicolumn{4}{|c|}{ Growth on colorants } & \multicolumn{2}{|c|}{ Agglutination with serum } \\
\hline & & & & & Thionine $20 \mu \mathrm{g}$ & Thionine $10 \mu \mathrm{g}$ & Basic fuchsin $20 \mu \mathrm{g}$ & Safranin $100 \mu \mathrm{g}$ & $A$ & $M$ \\
\hline SHB-Cam-Nor-152 & Ec-ClZ-Hum1 & + & + & + & - & - & + & + & - & + \\
\hline SHB-Ay-10 & Ec-ClZ-Hum2 & + & + & + & - & - & - & - & - & + \\
\hline SHB-Zon-Nor-370 & Ec-ClZ-Hum3 & + & + & + & + & - & + & + & - & + \\
\hline $\mathrm{B} 2 \dagger$ & & + & + & + & - & - & - & - & + & - \\
\hline B9‡ & & + & - & + & + & + & + & + & - & + \\
\hline
\end{tabular}

$\mathrm{ClZ}=$ International Centre for Zoonoses

${ }^{*}$ Blood culture.

† Control Brucella abortus biotype 2.

$\mp$ Control B. abortus biotype 9 .

knowledge about the true disease status of individuals. The method allowed for the incorporation of prior knowledge about prevalence of human brucellosis, test sensitivities, and specificities based on previous studies or expert opinions and the estimation of the dependence between the three tests conditional on the true disease status. Different models were constructed based on different combinations of the dependencies between the tests. Using appropriate model diagnostic tools such as the DIC, $p D$, and Bayesian $P$-values, it was demonstrated that the models incorporating the different covariance structures did not perform significantly better that the CID model. However, the model with CD among noninfected individuals was chosen for completeness. In addition, as explained in the introduction, the CD between tests is the most plausible biological hypothesis taking into account the immunological effectors that are implicated in each test. ${ }^{12,13}$

The preference for the model 9 (CD for noninfected cases) is related to the appraisal of several quality criteria $(p D, D I C$, and Bayes-p), but there appears to be no explicit biological explanation for this preference and its implications. It indicates that if the three tests in question (i-ELISA, SAT-EDTA, and RB) are applied to a noninfected person and if a first test is negative, the two other test(s) also tend to remain negative. Equivalently, if the first test is positive (i.e., a false-positive serological reaction), the other tests also have a tendency to be positive. This finding was previously demonstrated for other species. ${ }^{12}$ Conversely, in an infected person, if one test result is positive, the results for the other tests are not necessarily positive because the stage of infection of the patient becomes important (early infection mostly with presence of lgM versus later infection mostly with presence of $\lg G$ ). Implications for medical personnel include the importance of a proper anamnesis and the use of a multi-testing for a correct interpretation of the serological results in brucellosis-suspected patients.

In the present study, an overall seroprevalence of $1.88 \%(\mathrm{Cl}$ : 1.48-2.38) (70 in 3,733) was found in the entire study region, which is in sharp contrast with the official data of the Ecuadorian MSP that is only 67 cases between 2003 and 2007 (on a population of 12 million). The true prevalence was however estimated to be $1 \%$ (Cr. I.: $0.4-1.6){ }^{11}$ The results of the present investigation, of previous studies and the observations described by Ron-Roman et al., ${ }^{34}$ indicate a serious underreporting of human brucellosis in Ecuador and a rather high seroprevalence of this zoonosis in the rural population involved in animal production. In several American countries, human brucellosis does not seem to be very important, yet the reported figures may not reflect the real situation. The difficulties related to the diagnosis and the often ambiguous or even absent clinical symptoms are probably the principal reasons for the subnotification. ${ }^{33,35}$ The highly variable clinical reactions due to brucellosis, contributing to the difficulties in recognizing the disease, ${ }^{8,3}$ were also noted in the present study and underline the need for reliable diagnostic tests. ${ }^{1}$

The high concordance observed between the results of the three different tests $(\geq 0.96)$ is probably because the same effectors (IgG1 and/or lgM) are detected by several tests. Therefore, it is expected that tests are biologically conditional dependent. $^{12,13}$

The use of the Bayesian approach is incomplete without an assessment of variations in the prior information and the data

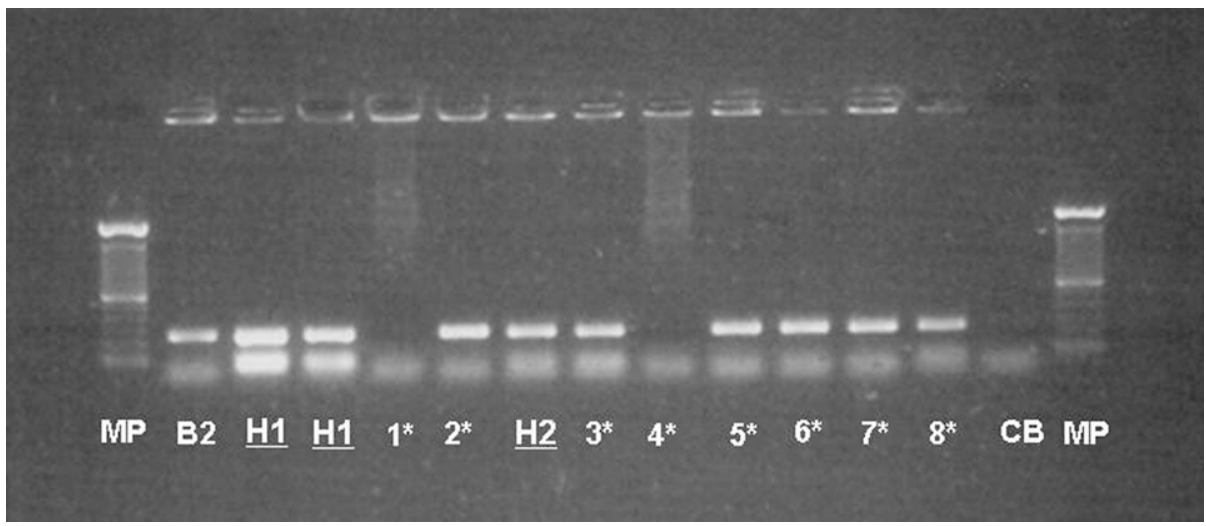

FIGURE 2. PCR-bscp31 of Brucella from blood cultures, isolated from positive persons. $\mathrm{B} 2=$ control Brucella abortus biotype 2; CB $=\mathrm{Blank}$ control; $\mathrm{ClZ}$ = International Centre for Zoonoses; $\mathrm{H} 1$ = Human sample 1 (Ec-ClZ-Hum-1); H2 = Human sample 2 (Ec-ClZ-Hum-2); MP = Molecular weight marker (100 bp AND Ladder $0.5 \mu \mathrm{g} / \mathrm{lane} 2 \%$ agarose gel stained). *Samples from complementary studies at ClZ. 


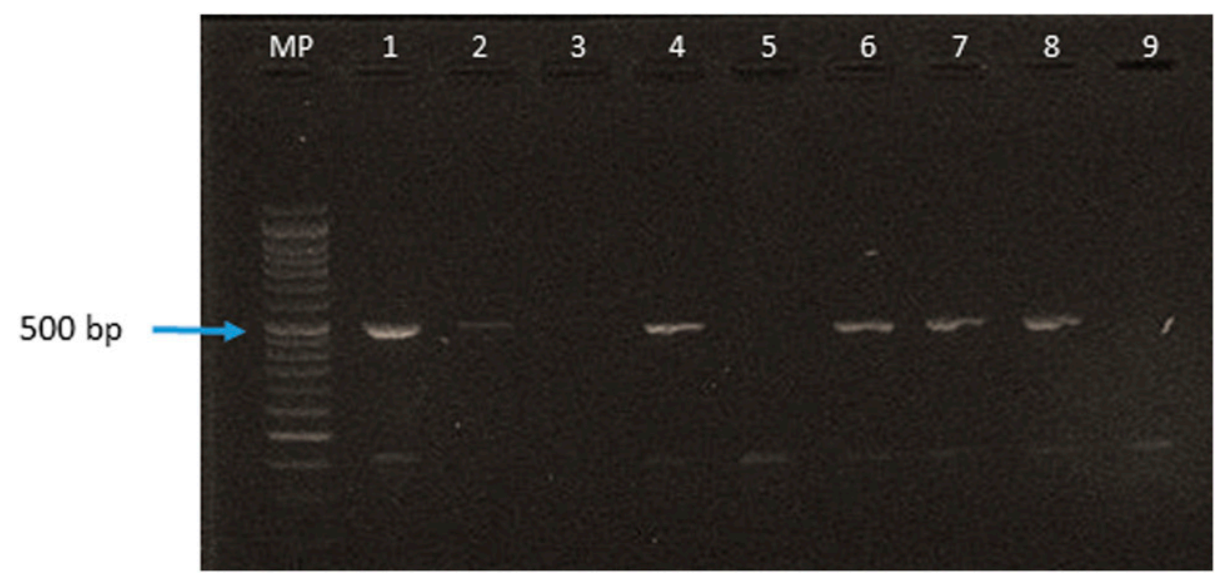

FIGURE 3. PCR-AMOS of Brucella abortus biovar 4 circulating in northwest Ecuador. AMOS PCR of samples of Brucella spp. MP $=$ Molecular Weight Marker. Line 1 = sample EC-CIZ-Bov-18. Line 2 shows EC-CIZ-Bov-15. Lines 4 and $6=$ bovine samples. Line 7 and $8=$ samples EC-CIZHum-3. Line 9 = negative control. $\mathrm{ClZ}=$ International Centre for Zoonoses. This figure appears in color at www.ajtmh.org.

on the estimated parameters using a sensitivity analysis. ${ }^{30,32,36}$ Sensitivity analyses indicated that the use of non-informative or lowly informative priors had no significant influence on the estimated prevalence and test sensitivities and specificities. It was also found that replacing the zero frequencies with one's yielded estimates that were similar to those based on the original data. This in turn suggests that the results of our study were dominated by the data as compared with the prior information, and thus are reliable.

The causal agent, that is, $B$. abortus biotype 4, was isolated and characterized in (apparently healthy) patients with high levels of IgM antibodies. Biotyping Brucella is important for the epidemiological knowledge because it can reveal geographical characteristics and allow a better understanding of the spread of the disease. ${ }^{37}$ Unfortunately, isolating and typing of Brucella spp. is not always possible because it requires high biosecurity laboratories and trained personnel. Furthermore, the low number of successful isolations in the present study is partly because of the localization of the bacteria in specific tissues and organs such as the bone marrow, cerebrospinal fluid (CSF), liver, kidneys, and spleen, which renders isolation from blood very unlikely. ${ }^{9,30}$

In the present study, $24.29 \%$ of the persons with a positive seroreaction showed no symptoms at all (Table 6). This is lower than the $45.6 \%$ reported by Perez et al. ${ }^{38}$ and the $99 \%$ found in a retrospective study of the symptomatology by Hernández-Bastida et al. ${ }^{39}$ This absence of typical symptoms is obviously one of the reasons for underreporting and the lack of data about the disease. ${ }^{3}$

$$
\text { TABLE } 6
$$

Human brucellosis: symptoms and frequency within the seropositive group $(n=70)$

\begin{tabular}{lcc}
\hline \multicolumn{1}{c}{ Symptoms } & Positive cases & Percentage \\
\hline Muscular pain & 29 & 41.43 \\
Joint pain & 25 & 35.71 \\
Fever & 17 & 24.29 \\
Debility & 17 & 24.29 \\
Headache & 16 & 22.86 \\
Nocturnal sweating & 13 & 18.57 \\
Cardiac problems & 10 & 14.29 \\
Anorexia & 4 & 5.71 \\
Insomnia & 4 & 5.71 \\
No symptoms & 17 & 24.29 \\
\hline
\end{tabular}

The absence of a National Policy and differential diagnostic tests hinders the development of surveillance and control programs in high-risk areas. It is thus difficult to have a realistic idea about incidence of the disease. In the past, little attention was given to brucellosis in Ecuador and it is necessary to develop programs to control (and eventually eradicate) brucellosis in the identified risk areas whereby highly sensitive diagnostic methods will be used both for humans and for animals with the objective of obtaining an early warning system and to determine the correct prevalence at national level.

Ecuador cannot be considered an endemic country for human brucellosis based on reported data ${ }^{40,41}$ although the results of the present study, based on factual data, prove quite the opposite. Underreporting and the absence of an active epidemiological vigilance may be the main reason for underestimation in supposedly brucellosis-free regions.

Finally, it is of utmost importance that evidence-based information be given to national and international donor organizations involved with future prevention, control, and research programs on brucellosis.

Received July 29, 2018. Accepted for publication January 10, 2019. Published online April 29, 2019.

Note: Supplemental appendices appear at www.ajtmh.org.

Acknowledgments: We thank the patients for their willingness to participate in this study.

Financial support: This work was supported by the Belgian Cooperation in the framework or the Institutional Collaboration between the Institute of Tropical Medicine in Antwerp, Belgium, and the International Centre for Zoonoses (ClZ) of Central University of Ecuador.

Disclaimer: The views expressed in this article are those of the authors and do not necessarily reflect the official policy or position of the Central University of Ecuador. Before being included in the study, participants were asked for verbal and written consent.

Authors' addresses: Jorge Ron-Román, International Center for Zoonoses, Central University of Ecuador, Ciudadela Universitaria, Quito, Ecuador, and Departamento de Ciencias de la Vida y la Agricultura, Carrera de Ingeniería Agropecuaria, Universidad de las Fuerzas Armadas - ESPE, Sangolquí, Ecuador, E-mail: jwron@ espe.edu.ec. Lenin Ron-Garrido, Maritza Celi-Erazo, Laura Vizcaíno Ordóñez, Jaime Calva-Pacheco, Pablo González-Andrade, and Washington Benítez-Ortíz, International Center for Zoonoses, Central University of Ecuador, Ciudadela Universitaria, Quito, Ecuador, E-mails: leninron@agro.uba.ar, mceli@uce.edu.ec, lauravizcainoord@ 
outlook.com, jimmyfran2000@yahoo.com, pdgonzalezandrade@ gmail.com, and wbenitez@uce.edu.ec. Emmanuel Abatih, Dirk Berkvens, and Jef Brandt, Department of Biomedical Sciences, Institute of Tropical Medicine, Antwerpen, Belgium, E-mails: emmanuel.abatih@ugent.be, dberkvens@itg.be, and jbrandt@ telenet.be. David Fretin, Department of Bacteriology and Immunology, Veterinary and Agrochemical Research Centre, Brussels, Belgium, E-mail: david.fretin@sciensano.be. Claude Saegerman, Research Unit of Epidemiology and Risk Analysis Applied to Veterinary Sciences (UREAR), Department of Infections and Parasitic Diseases, Faculty of Veterinary Medicine, University of Liege, Liège, Belgium, E-mail: claude.saegerman@uliege.be.

Reprint requests: Jorge Ron-Román, International Center for Zoonoses, Central University of Ecuador, P.O. Box 17-03-100, Quito, Ecuador, E-mail: jwron@espe.edu.ec, Fax: +59322904801.

\section{REFERENCES}

1. Young EJ, 1997. Especies de brucella. Mandell G, Bennett J, Dolin $\mathrm{R}$, eds. Enfermedades Infecciosas, Principios y Practica. Buenos Aires, Argentina: Editorial Medica Paramericana SA, 2300-2320.

2. Orduña A et al., 2000. Evaluation of an immunocaptureagglutination test (Brucellacapt) for serodiagnosis of human brucellosis. J Clin Microbiol 38: 4000-4005.

3. Saegerman C, Berkvens D, Godfroid J, Walravens K, 2010. Bovine brucellosis. Lefèvre P, Blancou J, Chermette R, Uilenberg G, eds. Infectious and Parasitic Disease of Livestock. Cachan, France: Lavoisier, 991-1021.

4. Corbel MJ, 2006. Brucellosis in Humans and Animals. Geneva, Switzerland: World Health Organization.

5. Torres $\mathrm{H}, 2008$. Control de Brucelosis bovina-programa nacional. Minist Agric Ganad Acuacultura y Pesca (MAGAP), Serv Ecuatoriano Saniadad Agropecu (SESA) Ed. Quito, Ecuador, 30.

6. Bowden R, 1996. Temas de Microbiología Veterinaria. Stanchi B, Martino P, Gentilini E, Reinoso E, eds. Inter-Medica, Buenos Aires, Argentina: Sur. Primera, 159-175.

7. Saegerman C, Berkvens D, Godforid J, Walravens K, 2010. Chapter 77: bovine brucellosis. Infectious and Parasitic Disease of Livestock. Paris, France: Lavoisier et Commonwealth Agricultural Bureau - International, 991-1011.

8. Sarguna P, Bilolikar AK, Rao A, Mathur DR, 2002. Brucellosis in association with HIV infection-a case report. Indian $\mathrm{J}$ Med Microbiol 20: 221-222.

9. Doganay M, Aygen B, 2003. Human brucellosis: an overview. Int $J$ Infect Dis 7: 173-182.

10. Díaz R, Leiva J, Rubio M, Dorronsoro L, 2001. Diagnóstico de la brucelosis humana. Díaz E, Hernández L, Valero G, Arellano B, eds. Diagnóstico de Brucelosis Animal. México: INIFAP, 198-212.

11. INEC, 2008. Anuario de estadísticas hospitalarias. Informe Analítico. Quito, Ecuador: INEC, 92.

12. Saegerman $C$ et al., 2004. Evaluation of three serum i-ELISAs using monoclonal antibodies and protein $G$ as peroxidase conjugate for the diagnosis of bovine brucellosis. Vet Microbiol 100: 91-105.

13. Sanogo M, Thys E, Achi YL, Fretin D, Michel P, Abatih E, Berkvens $D$, Saegerman C, 2013. Bayesian estimation of the true prevalence, sensitivity and specificity of the Rose Bengal and indirect ELISA tests in the diagnosis of bovine brucellosis. Vet J 195: 114-120.

14. Dendukuri N, Joseph L, 2001. Bayesian approaches to modeling the conditional dependence between multiple diagnostic tests. Biometrics 57: 158-167.

15. Branscum AJ, Gardner IA, Wagner BA, Mclnturff PS, Salman MD, 2005. Effect of diagnostic testing error on intracluster correlation coefficient estimation. Prev Vet Med 69: 63-75.

16. Berkvens D, Speybroeck N, Praet N, Adel A, Lesaffre E, 2006. Estimating disease prevalence in a Bayesian framework using probabilistic constraints. Epidemiology 17: 145-153.

17. Vacek PM, 1985. The effect of conditional dependence on the evaluation of diagnostic tests. Biometrics 41: 959-968.

18. Godfroid J, Boelaert F, 1995. Prescriptions Pour le Diagnostic Sérologique de la Brucellose. Uccle, Belgium: CODA-CERVA, 47.
19. Baily GC, Kraahn JB, Drasar BS, Stokeer NG, 1992. Detection of Brucella melitensis and Brucella abortus by DNA amplification. J Trop Med Hyg 95: 271-275.

20. Navarro E, Escribano J, Fernández JA, Solera J, 2002. Comparison of three different PCR methods for detection of Brucella spp. in human blood samples. FEMS Immunol Med Microbiol 34: 147-151.

21. Bricker BJ, Halling SM, 1994. Differentiation of Brucella abortus bv. 1, 2, and 4, Brucella melitensis, Brucella ovis, and Brucella suis bv. 1 by PCR. J Clin Microbiol 32: 2660-2666.

22. Spiegelhalter DJ, Best NG, Carlin BP, van der Linde A, 2002. Bayesian measures of model complexity and fit. $J R$ Stat Soc Ser B 64: 583-639.

23. Nérette P, Stryhn H, Dohoo I, Hammell L, 2008. Using pseudogold standards and latent-class analysis in combination to evaluate the accuracy of three diagnostic tests. Prev Vet Med 85: 207-225.

24. Kadri SM, Rukhsana A, Laharwal MA, Tanvir M, 2000. Seroprevalence of brucellosis in Kashmir (India) among patients with pyrexia of unknown origin. J Indian Med Assoc 98: 170-171.

25. Araj GF, Azzam RA, 1996. Seroprevalence of brucella antibodies among persons in high-risk occupation in Lebanon. Epidemiol Infect 117: 281-288.

26. Rahman AK, Dirk B, Fretin D, Saegerman C, Ahmed MU, Muhammad N, Hossain A, Abatih E, 2012. Seroprevalence and risk factors for brucellosis in a high-risk group of individuals in Bangladesh. Foodborne Pathog Dis 9: 190-197.

27. Gómez MC, Nieto JA, Rosa C, Geijo P, Escribano MA, Muñoz A, López C, 2008. Evaluation of seven tests for diagnosis of human brucellosis in an area where the disease is endemic. Clin Vaccine Immunol 15: 1031-1033.

28. Memish ZA, Almuneef M, Mah MW, Qassem LA, Osoba AO, 2002. Comparison of the Brucella standard agglutination test with the ELISA IgG and IgM in patients with Brucella bacteremia. Diagn Microbiol Infect Dis 44: 129-132.

29. Gelman A, Rubin DB, 1992. Inference from iterative simulation using multiple sequences. Stat Sci 7: 457-472.

30. Haley C, Wagner B, Puvanendiran S, Abrahante J, Murtaugh MP, 2011. Diagnostic performance measures of ELISA and quantitative PCR tests for porcine circovirus type 2 exposure using Bayesian latent class analysis. Prev Vet Med 101: 79-88.

31. Praud A, Gimenez O, Zanella G, Dufour B, Pozzi N, Antras V, Meyer L, Garin-Bastuji B, 2012. Estimation of sensitivity and specificity of five serological tests for the diagnosis of porcine brucellosis. Prev Vet Med 104: 94-100.

32. Enøe C, Georgiadis MP, Johnson WO, 2000. Estimation of sensitivity and specificity of diagnostic tests and disease prevalence when the true disease state is unknown. Prev Vet Med 45: 61-81.

33. Robinson A, 2003. Guidelines for Coordinated Human and Animal Brucellosis Surveillance. Rome, Italy: FAO.

34. Ron-Roman L, Benitez W, Speybroeck N, Ron J, Saegerman C, Berkvens D, Abatih E, 2013. Spatio-temporal clusters of incident human brucellosis cases in Ecuador. Spat Spatiotemporal Epidemiol 5: 1-10.

35. Serra Alvarez J, Godoy García P, 2000. Incidence, etiology and epidemiology of brucellosis in a rural area of the province of Lleida [in Spanish]. Rev Esp Salud Publica 74: 45-53.

36. Caraguel C, Stryhn H, Gagné N, Dohoo I, Hammell L, 2012. Use of a third class in latent class modelling for the diagnostic evaluation of five infectious salmon anaemia virus detection tests. Prev Vet Med 104: 165-173.

37. Roux J, 1979. Epidemiology and prevention of brucellosis [in French]. Bull World Health Organ 57: 179-194.

38. Pila-Pérez R, Pila-Peláez R, Paulino-Basulto M, Hernández-Pupo O, Gacía-Peña JG, del Sol-Torres G, 1997. Estudio clínico de la brucelosis humana. Rev Med Urug 13: 110-117.

39. Hemández-Bastida A, García-Ramírez P, Cruz-Estrada A, Rojo J, 1999. Seroprevalencia de brucelosis en disponentes de sangre del Hospital General de México. Rev Médica Hosp Gen México 62: 107-112.

40. Ron-Román J, Saegerman C, Minda-Aluisa E, Benítez-Ortíz W, Brandt J, Douce R, 2012. First report of orchitis in man caused by Brucella abortus biovar 1 in Ecuador. Am J Trop Med Hyg 87: 524-528.

41. Pappas G, Papadimitriou P, Akritidis N, Christou L, Tsianos EV, 2006. The new global map of human brucellosis. Lancet Infect Dis 6: 91-99. 